\section{EXPERIENCES OF FAMILIES WITH CHILDREN IN BRISTOL, UNITED KINGDOM WITH NEURODISABILITY DURING THE COVID 19 PANDEMIC RESTRICTIONS}

Alexandra Nettleton, Caroline Bodey. UK

\subsection{6/bmjpo-2021-RCPCH.50}

Background Due to the COVID 19 pandemic from March 2020 until June 2020 the United Kingdom was under a National lockdown, with many health services and allied health professional services significantly impacted. We wanted to capture the experiences on children with disabilities and their families during this time.

Objectives

1. To assess the effects of the restrictions due to the COVID19 pandemic on children with disabilities and their families.

2. Improve our understanding of the issues faced to enable us to support them.

Methods Telephone questionnaire interviews to eleven parents of children under the care of community child health with a neurodevelopmental disability.

Results Children's access to therapy was significantly impacted with the majority of children not receiving their usual therapy. Access to the GP was reportedly more difficult and hospital appointments delayed and cancelled. Half of families reported medication being more difficult to get hold of. Respite care was unavailable. Families report being negatively impacted by through exhaustion and concern about their child's health needs not being met.

The majority of the children did not attend education during the pandemic despite being in the vulnerable category. Some reported no contact from educational during this time.

Most families reported restrictions negatively impacted on their child's physical health. Some didn't take their children out for the duration of lockdown.

Changes in emotional well being of the children were reported ( appetite, disruptive behaviour, toileting regression, sleep regression, worsening spasticity and anxiety). Adults were emotionally affected by the restrictions (increased arguments, exhaustion, juggling increased demands and loneliness). Positives were also reported (more family time).

Access to support was minimal with only one family accessing emotional support. There were very mixed feeling about preference for clinical appointments including telephone, face to face, home visits and video links. Concerns were raised about fears of professionals in masks, language barriers worsened over the telephone and children struggling to engage with video consultations.

Conclusions The children on our caseload are vulnerable for a variety of reasons and require input from a range of multi professionals to meet their diverse needs.

The covid 19 restrictions had a negative impact not only on the services our children received but on their emotional and physical health and on the well-being of their families.

\section{VITAMIN B2 (RIBOFLAVIN) AS PROPHYLAXIS FOR MIGRAINES IN CHILDREN: A RETROSPECTIVE REVIEW}

Annie John, Wasiullah Shinwari. UK

10.1136/bmjpo-2021-RCPCH.51
Background Migraines represent a substantial burden of disease in childhood, occurring in $3-10 \%{ }^{1}$ of school aged children. These are associated with increased absences from school and subsequent academic sequlae. While, the pathophysiology is not yet fully understood, it has been found that sufferers of migraines show insufficiencies in mitochondrial energy processing and thus in energy metabolism in the brain. ${ }^{2}$ Riboflavin or vitamin B2 acts as a catalyst in the action of flavoenzymes and plays a vital role in the electron transport chain. ${ }^{2}$ It has therefore been postulated that supplementing this could act to improve energy production and act as prophylaxis for migraines ${ }^{2}$.

Current National Institute for Clinical Excellence (NICE) guidelines suggests Riboflavin supplement to be considered in children over 12 for migraine proplylaxis. Evidence in adult populations has been promising with a small number of studies demonstrating the reduction of migraine frequency and length. ${ }^{3-7}$ Studies in children however are scarce with mixed result.

Objectives To review the effectiveness of Riboflavin as prophylaxis for Migraines in children under 18 years.

Methods Cases of migraines in children who were managed with Riboflavin at Worcester Royal Hospital were identified retrospectively. Patient notes were subsequently reviewed.

Results 21 children were identified with ages ranging from 9 to 17 years (median 14). There were equal numbers of males and females. 19 (90\%) patients were diagnosed with Migraine and $2(10 \%)$ with non-migranous headaches, following appropriate investigations. The most frequent associated symptom was nausea, followed by visual disturbance/photophobia at $62 \%(13)$ and 43\% (9) respectively.

All patients received a 3-month course of Riboflavin 400 mg once daily, bar 1 under the age of 10 who received 200 mg once daily. $12(57 \%)$ patients were also provided with Sumatriptan and Cyclizine for acute attacks. 8 (38\%) patients had previously failed to see symptomatic improvement with other prophylactic medications. Follow up was between 4 and 28 weeks (median 13 weeks). Follow up information was not available for 2 children.

$17 / 19$ (89\%) patients reported significant improvement in migraine symptoms. Of these, $7 / 19$ (37\%) patients reported complete resolution of symptoms. No adverse effects were reported.

Conclusions $200-400 \mathrm{mg}$ a day of Riboflavin is shown to be an effective prophylactic medication in children with Migraines in this review, with both improvement and resolution reported.

Current available evidence in children is limited with mixed results. 2 randomised control trials (RCTs) with $\mathrm{n}=42^{10}$ and $\mathrm{n}=48^{11}$, both of which used doses of $50 \mathrm{mg} /$ day-200 mg/day, failed to show a difference in treatment and placebo groups. 2 further RCTs with $\mathrm{n}=98^{13}$ and $\mathrm{n}=90^{8}$, and a retrospective study without control $(n=41)^{9}$ showed clinical improvement in groups given $200-400 \mathrm{mg} / \mathrm{day}$ of Riboflavin. This mirrors the positive results seen in this review suggesting improvement in children may be dose related. Adverse effects reported included vomiting, diarrhoea and urinary frequency.

Limitations Due to retrospective element, this review does not include a control group and it not been possible to quantify improvement. The sample size is small.

Further study with randomised control trials and a larger sample size using higher doses of Riboflavin is warranted. 